Given that eggs are so problematic, some teams are attacking the problem of reprogramming from a different angle. They are trying to see whether other kinds of cells share an egg's ability to reprogramme a nucleus. One such candidate is an embryonic stem cell itself. Recently, Harvard University researcher Kevin Eggan and his colleagues transformed adult body cells to an embryonic state by fusing them with embryonic stem cells ${ }^{10}$. And some experiments have even suggested that embryonic stem cells might be better at certain aspects of reprogramming than oocytes.

But the major drawback of this method is that the chromosomes of the embryonic stem cell used to spark the process are retained. This limits a cell's therapeutic potential because a patient's immune system could recognize the leftover chromosomes and launch an attack. Researchers are working on fixes, however. For example, Paul Verma from Monash University has devised a way of getting rid of the unwanted chromosomes ${ }^{11}$, and now has unpublished evidence that mouse cells might be reprogrammed using this approach.

Others are searching for those seemingly magical factors in eggs that allow them to wind an adult nucleus back to an embryonic form. Nobuaki Kikyo of the Stem Cell Institute at the University of Minnesota in Minneapolis, for example, has fished out factors from frog eggs that can repackage chromosomes, dismantle the nucleus's structure and switch on gene activity - all key aspects of reprogramming ${ }^{12}$. But this approach will take time. "Someone might get lucky, but I think it's a long way off," says Keith Latham from Temple University School of Medicine in Philadelphia, Pennsylvania.

There is unlikely to be one single way to mimic the almost mystical reprogramming ability of a human egg. The answer, says Weissman, could be to combine methods - kickstarting the process with one approach, and finishing it with another.

Carina Dennis is Nature's Australasian correspondent.

1. Cyranoski, D. Nature 439, 122-123 (2006).

2. Lavoir, M.-C., Weier, J., Conaghan, J. \& Pedersen, R. A. Reprod. Biomed. Online 11, 740-744 (2005).

3. Stojkovic, M. et al. Reprod. Biomed. Online 11, 226-231 (2005).

4. Cibelli, J. B. et al. J. Regen. Med. 2, 25-31 (2001)

5. O'Brien, M. J., Pendola, J. K. \& Eppig, J. J. Biol. Reprod. 68, 1682-1686 (2003).

6. Biron-Shental, T. et al. Fertil. Steril. 81, 716-719 (2004).

7. Hübner, K. et al. Science 300, 1251-1256 (2003)

8. Clark, A. T. et al. Hum. Mol. Genet. 13, 727-739 (2004)

9. Chen, Y. et al. Cell Res. 13, 251-263 (2003).

10. Cowan, C. A., Atienza, J., Melton, D. A. \& Eggan, K. Science 309, 1369-1373 (2005)

11. Pralong, D. et al. Cloning Stem Cells 7, 265-271 (2005)

12. Tamada, H. et al. Mol. Cell. Biol. 26, 1259-1271 (2006).

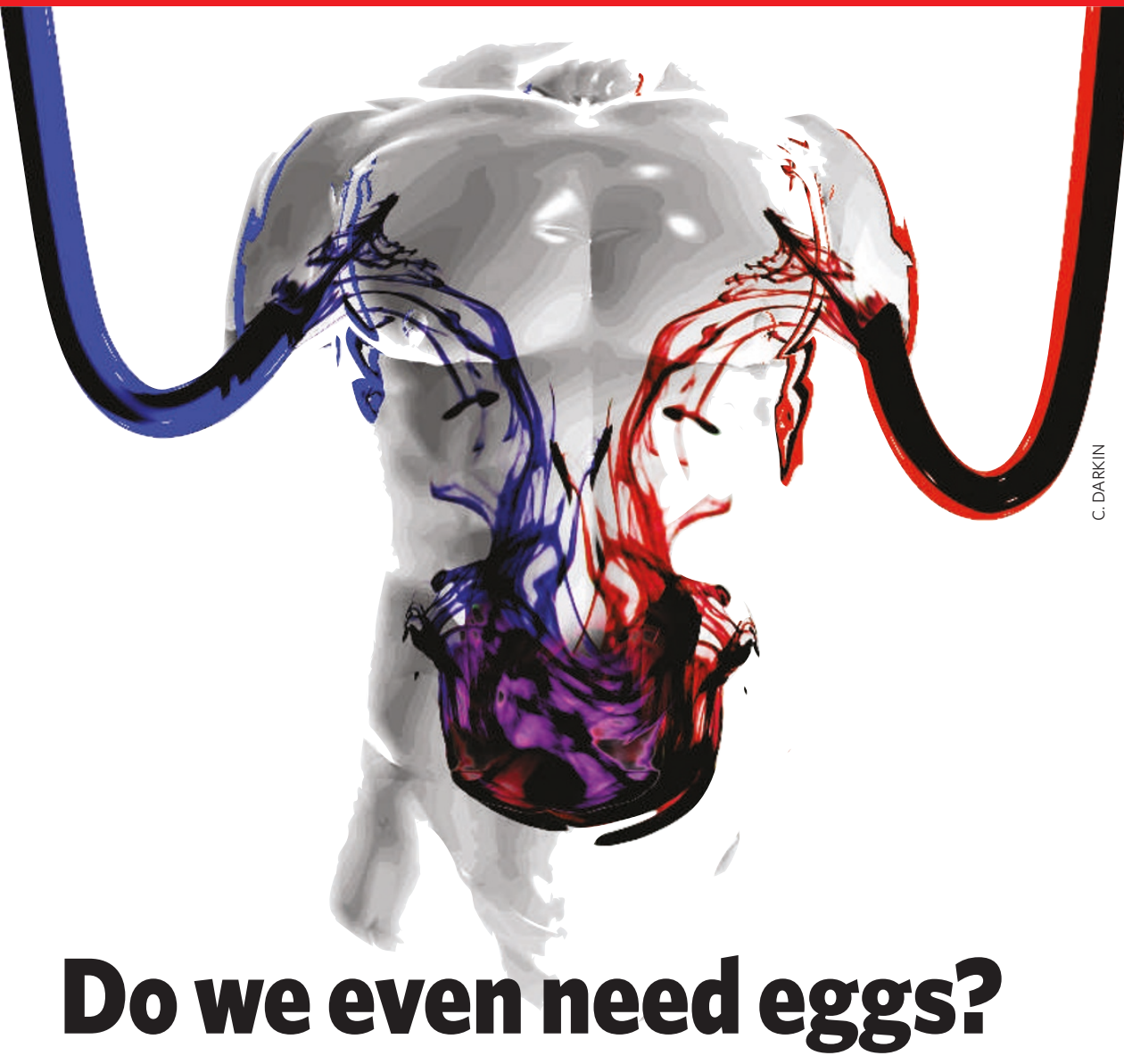

obody likes rejection, but for a transplant patient it can be a death sentence. The risk that a patient's immune system will see a transplanted organ, or graft, as 'foreign' rather than 'self', forces transplant patients on to immunosuppressant drugs that can have severe side effects. Therapeutic cloning, its enthusiasts say, could solve the problem by allowing doctors to grow cells and tissues that are perfectly matched to individual patients. In this approach, a patient's DNA is transferred into an egg which is persuaded to develop into stem cells that in turn generate spare-part tissues. But many researchers now think therapeutic cloning is unrealistic, largely owing to the scarcity of human eggs.

So the spotlight is turning on to different strategies, aimed at persuading the immune system to tolerate foreign tissue. "The field is moving very fast," says Harry Moore of the Centre for Stem Cell Biology at the University of Sheffield, UK. "Ten years ahead there may be no need for cloning, except in certain cases."

There are different ways to increase the success of tissue transplants. One is to develop generic stem cells, cell lines and tissues, and then persuade the immune system to accept them. These could be therapeutic transplants of, say, insulinproducing cells to treat

"Ten years ahead there may be no need for cloning." - Harry Moore diseases such as type 1 diabetes. Those championing this idea admit it is years, or decades, from the clinic. So more pragmatic approaches are also under development. Instead of trying to make the immune system perfectly tolerant of a transplant, some researchers are aiming to increase its tolerance enough for patients to sharply reduce their dependence on powerful immunosuppressive drugs.

At the moment, most people who have an organ transplant face a lifetime of treatment with drugs that affect the whole immune system, such as cyclosporine and steroids. Although these drugs increase the life of a grafted organ by several years, they often fail to prevent its eventual rejection, and they put patients at risk of infections, cancers and kidney failure.

\section{Drug problem}

In studies on small numbers of patients who have had organ transplants, medical teams are discovering that they can manipulate the immune system so that drug treatment can be reduced. For example, Chris Watson and Roy Calne at Addenbrooke's Hospital, Cambridge $^{1}$, are among those who gave patients an antibody, Campath-1 (alemtuzumab), at the time of an organ transplant. This antibody depletes lymphocytes, a large family of key immune cells.

Watson and Calne then gave lower doses of immunosuppressive drugs to the patients than would normally be given following a transplant, and no steroids. For the five-year study period, the patients' grafts survived as 
well as did those in patients given conventional treatment. This treatment approach is not ready for widespread use, says Herman Waldmann of the University of Oxford, UK, who developed Campath-1. But, he adds, it demonstrates the principle that the use of immunosuppressive drugs can be minimized.

Although researchers disagree about exactly how the antibody treatment works, some speculate that by holding off an immune attack, it somehow gives the immune system time to 'learn' to tolerate the graft. The aim now is to understand more about how this tolerance develops, so that more precise therapies can be designed. After all, says Waldmann, an antibody that blocks whole classes of lymphocytes is still a relatively crude instrument.

The immune system distinguishes between 'self' and 'non self' proteins because of the actions of a subset of lymphocytes called $\mathrm{T}$ cells. Each T cell carries a receptor on its surface, uniquely shaped to fit a specific protein fragment or peptide. Other cells of the immune system patrolling the body pick up proteins, or antigens, and present fragments to the T cells. If a fragment fits into a specific $\mathrm{T}$-cell receptor, it acts rather like a key in a lock, and, given certain conditions, will switch the $\mathrm{T}$ cell into an active state. The $\mathrm{T}$ cell then coordinates an immune response against that protein.

Of course, if the T cells became activated in response to self proteins, disaster would ensue. So when new T cells maturing in the thymus (an organ just above the heart) encounter a range of protein fragments,
T cells that specifically recognize self antigens are killed off or 'deleted'. This editing, known as central tolerance, is what usually protects us against autoimmune disease. The process happens mainly in infancy and youth, with the thymus shrinking as an individual reaches maturity.

But while this central tolerance is developing, the body can be fooled into accepting foreign cells as self - at least in newborn mice ${ }^{2}$. The young animals are given infusions of bone-marrow cells. These cells are the precursors of T cells and dendritic cells, another type of lymphocyte that plays a key role in presenting protein frag-
"The important thing is to take the field onwards in all the areas with potential." - Herman Waldmann its place. The presence of the foreign bonemarrow cells creates a state known as 'mixed chimaerism' ${ }^{\prime 3}$, where $T$ cells that react to either host- or donor-derived antigens are killed off, allowing the animal to accept other tissue from the same donor.

Megan Sykes, David Sachs and colleagues have tried to harness this phenomenon of chimaerism to persuade the immune system to accept transplants of bone marrow and kidneys in a small number of adult patients. They treated the patients with drugs to deplete the host lymphocytes, then transplanted the new tissues. At first, the patients' blood appeared chiments to T cells. When the dendritic cells derived from this foreign bone marrow migrate to the thymus and present their protein fragments to the $\mathrm{T}$ cells maturing there, the fragments are seen as self and any $\mathrm{T}$ cells that recognize them are killed off. As a result, the animals later accept grafts of skin from the same donor source.

\section{Layers of command}

But this approach is obviously not suitable for treatment in adult humans, because the thymus is producing so few new T cells. So researchers have tried to manipulate the mature immune system to achieve a sort of artificial central tolerance. In animal experiments, the host's bone marrow is partly inactivated and new donor bone marrow infused in maeric - that is, the researchers found various types of blood cells in it bearing the donor's signature. If these findings reflect the animal experiments, they would suggest that $T$ cells reactive to the donor tissues had been deleted as though they were targeting self.

But there was a puzzle. The grafts survived for years, yet, strangely, the donor blood cells faded away after about 12 weeks, suggesting the patients were no longer chimaeric. This suggested that chimaerism could not fully explain the survival of the grafts, but that something else must be helping to protect them from rejection. Sykes suggests that the kidney grafts themselves must be doing something that helps the host immune system tolerate them. Instead of involving the central deletion of $\mathrm{T}$ cells in the thymus, this form of

\section{GREAT EXPECTATIONS}

Millions of people around the world happily tolerate foreign tissues for months on end. No scientific trick here: simply pregnancy. Researchers are trying to understand how pregnant women tolerate their fetus in the hope of understanding tolerance, or even preventing miscarriage.

One such team is headed by Harry Moore at the University of Sheffield, UK. He and his team are studying a particular kind of bloodvessel cell found in the placenta. This secretes a protein known as HLA-G, which seems to help prevent the mother's T cells from attacking her fetus.

Recently, researchers found that a type of immune cell called a monocyte can also make HLA-G. Intriguingly, these cells infiltrate newly transplanted tissue. The higher the levels of HLA-G expressed, the greater the chance that the transplant will be accepted. This finding, says Moore, suggests that HLA-G may naturally help to protect some tissue grafts, although he stresses that no one knows how the process works.

Moore's group hopes that studies of this kind of blood-vessel cell may reveal more about how an embryo normally implants and why some mothers' immune systems do reject their fetuses. In the longer term, says Moore, it might be possible to develop the cells for transplant therapy in heart disease. The cells might also be given with other therapeutic tissues, with the aim of making the recipient tolerate both types of cell.

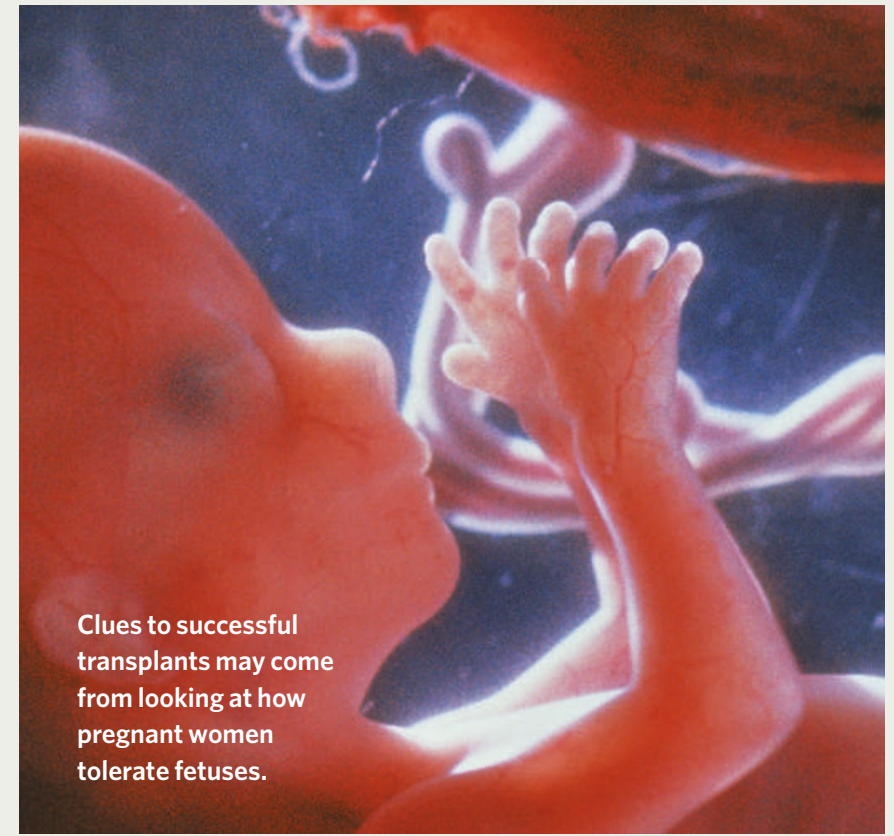


MAKING 'FOREIGN' LOOK LIKE 'SELF'

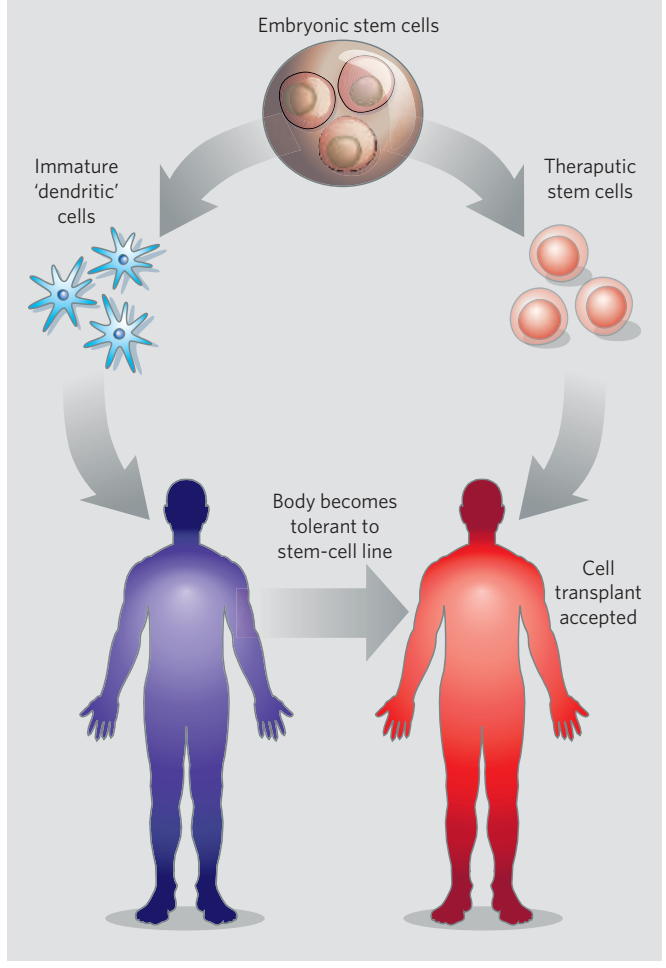

encounter. Instead they will either 'decommission' $\mathrm{T}$ cells altogether, or push them to become regulatory $\mathrm{T}$ cells, in which they police other $\mathrm{T}$ cells and suppress any attackers. Together with Paul Fairchild and others, Waldmann is trying to exploit dendritic cells as a tool to develop tolerance to stem-cell transplants.

Five years ago, Fairchild and his colleagues worked out how to make mouse embryonic stem cells differentiate into dendritic cells ${ }^{5}$ in a dish in the lab. He and his team found that their characteristics were stable over relatively long periods. The dendritic cells were fully differentiated, so could not reverse their development to become stem cells, although they had the 'immature' dendritic cell features - the

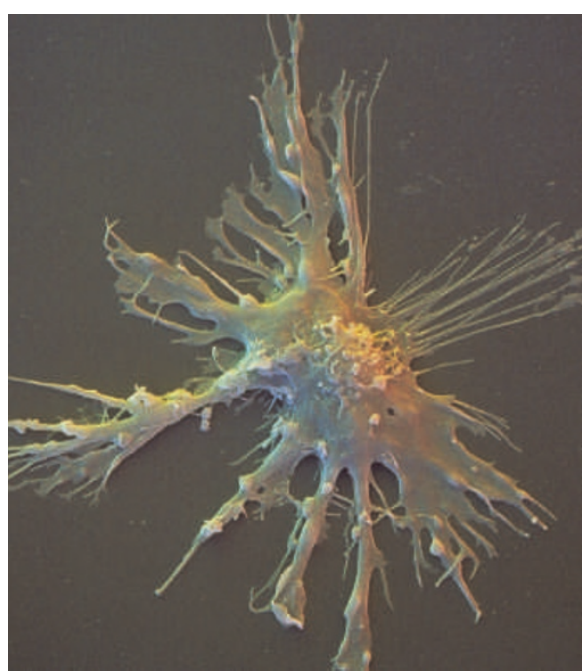

Dendritic cells could be harnessed as a tool to help promote tolerance of tissue transplants. form most likely to make T cells tolerant.

Fairchild reasoned that human dendritic cells, cultured in the same way, could play a role in inducing transplant tolerance. If the dendritic cells and the therapeutic stem cells required for a given transplant - say, heart-muscle cells - could both be cultured in lines from the same stem-cell source, the dendritic cells could be used to make the recipient tolerate the therapeutic cells $^{6}$ (see graphic).

\section{Early days}

Fairchild and his colleagues have yet to test this idea fully in mice, although their early unpublished experiments suggest that mice injected with dendritic cells carrying a specific protein can be made to accept skin grafts carrying the same protein. Fairchild has begun culturing human dendritic cells from embryonic stem cells.

There is, however, the risk that a dendritic cell could switch into its mature state, and so activate $T$ cells. If this were the case, the cells could help destroy, rather than protect, the graft. Fairchild and Waldmann are exploring the idea of treating the cells with various compounds in culture to keep them in their 'tolerizing' form ${ }^{7}$. But, as Fairchild stresses repeatedly, these are early days.

But not all researchers are confident that cells differentiated from embryonic stem cells will end up being suitable for transplant therapy. Mick Bhatia, an immunology researcher now at McMaster University in Ontario, Canada, worries that even under the most rigorous conditions, an undifferentiated stem cell could still slip in among the differentiated cells. Undifferentiated stem cells can trigger a form of cancerous growth. Waldmann and Fairchild agree that before the approach could be used for treatment, tests must be developed to identify any undifferentiated cells in a cultured line.

No one pretends that tolerance is going to arrive tomorrow. "But the important thing is to take the field onwards in all the areas with potential," says Waldmann. As degenerative diseases such as heart disease become more common, Fairchild and other researchers are convinced that they should intensify their efforts. "We've got a lot further to go, but it's very exciting."

Phyllida Brown is a science writer in Exeter, UK.
Watson, C. J. et al. Am. J. Transplant. 5, 1347-1353 (2005)
2. Billingham, R. et al. Nature $\mathbf{1 7 2 , 6 0 3 - 6 0 6 ~ ( 1 9 5 3 ) ~}$
3. Buhler, L. H. et al. Transplantation 74, 1405-1409 (2002).
4. Steinman, R. M. et al. Annu. Rev. Immunol. 21, 685 (2003)
5. Fairchild, P. J. et al. Curr. Biol. 10, 1515-1518 (2000).
6. Fairchild, P. J. et al. Trends Immunol. 25, 465-470 (2004).
7. Fairchild, P. J. et al. Intl. Immunopharmacol. 5, 13-21(2005). 